\title{
Holographic bulk viscosity: GPR vs EO
}

\author{
Alex Buchel, ${ }^{a, b}$ Umut Gürsoy ${ }^{c}$ and Elias Kiritsis ${ }^{d, e, 1}$ \\ ${ }^{a}$ Department of Applied Mathematics, University of Western Ontario, \\ London, Ontario N6A 5B\%, Canada \\ ${ }^{b}$ Perimeter Institute for Theoretical Physics, \\ Waterloo, Ontario N2J 2W9, Canada \\ ${ }^{c}$ Theory Group, Physics Department, CERN, \\ CH-1211 Geneva 23, Switzerland \\ ${ }^{d}$ Crete Center for Theoretical Physics, Department of Physics, University of Crete, \\ 71003 Heraklion, Greece \\ ${ }^{e}$ Laboratoire APC, Université Paris-Diderot Paris 7, CNRS UMR 7164, \\ 10 rue Alice Domon et Léonie Duquet, 75205 Paris Cedex 13, France \\ E-mail: abuchel@uwo.ca, umut.gursoy@cern.ch
}

ABSTRACT: Recently Eling and $\mathrm{Oz}$ (EO) proposed a formula for the holographic bulk viscosity, in arXiv:1103.1657, derived from the null horizon focusing equation. This formula seems different from that obtained earlier by Gubser, Pufu and Rocha (GPR) in arXiv:0806.0407 calculated from the IR limit of the two-point function of the trace of the stress tensor. The two were shown to agree only for some simple scaling cases. We point out that the two formulae agree in two non-trivial holographic theories describing RG flows. The first is the strongly coupled $\mathcal{N}=2^{*}$ gauge theory plasma. The second is the semi-phenomenological model of Improved Holographic QCD.

KEYwords: Gauge-gravity correspondence, Duality in Gauge Field Theories, AdS-CFT Correspondence

ARXIV EPRINT: 1104.2058

\footnotetext{
${ }^{1}$ http://hep.physics.uoc.gr/ kiritsis/.
} 


\section{Contents}

1 Introduction and summary 1

2 The GPR formula for the holographic bulk viscosity 3

2.1 The EO versus GPR formula for the bulk viscosity 4

3 Bulk viscosity calculation in the $\mathcal{N}=2^{*}$ plasma 4

3.1 The computation of $c_{11}^{-}$in $x$-gauge 5

$3.2 \mathcal{N}=2^{*}$ plasma at high temperatures $\quad 5$

$\begin{array}{lll}3.3 \mathcal{N}=2^{*} \text { plasma at generic temperatures for } \frac{m_{b}^{2}}{T^{2}}>0 & 7\end{array}$

4 Bulk viscosity calculation in improved holographic QCD $\quad 7$

4.1 Computation of $c_{11}^{-}$using phase variables 8

$\begin{array}{ll}4.2 \text { The fluctuation equation } & 9\end{array}$

$\begin{array}{ll}4.3 & \text { Numerical results for the holographic-QCD model } \\ \end{array}$

A $\frac{d A}{d \phi}$ in $\mathcal{N}=2^{*}$ plasma

$\begin{array}{ll}\text { B Coefficients } \mathcal{H}_{i} & 12\end{array}$

C Chamblin-Reall backgrounds $\quad 12$

\section{Introduction and summary}

The bulk viscosity of strongly coupled thermal systems is a quantity of phenomenological importance. On the other hand it is quite difficult to compute. The main difficulty arises from the fact that the bulk viscosity, as one of the plasma deep-infrared transport coefficients, is sensitive to the microscopic (ultraviolet) parameters of the theory. ${ }^{1}$ Necessarily, a computation of the bulk viscosity in a given system requires the understanding of its physics over a wide range of scales. It is perhaps not surprising that the first computation of the bulk viscosity in gauge theory plasmas [1] was performed in the framework of gauge theory/string theory correspondence $[2,3]$.

In [4] it was observed that for a large class of holographic models, the bulk viscosity of the strongly coupled plasma satisfies the following bound

$$
\frac{\zeta}{\eta} \geq 2\left(\frac{1}{3}-c_{s}^{2}\right)
$$

\footnotetext{
${ }^{1}$ The only exception is a conformal theory, where the scale invariance imposed the bulk viscosity to vanish.
} 
where $\eta$ is the universal shear viscosity of strongly coupled holographic plasma [5-8], and $c_{s}$ is the speed of sound waves in plasma. The computation of the bulk viscosity which led to (1.1) was based on analyzing the dispersion relation of the sound waves in the plasma. Alternatively, the bulk viscosity can be computed using the Kubo formula

$$
\zeta=-\frac{4}{9} \lim _{\omega \rightarrow 0} \frac{1}{\omega} \operatorname{Im} G_{R}(\omega),
$$

where $G_{R}$ is the retarded correlation function of the stress-energy tensor

$$
G_{R}(\omega)=-i \int d t d^{3} x e^{i \omega t} \Theta(t)\left\langle\left[\frac{1}{2} T_{i}^{i}(t, \vec{x}), \frac{1}{2} T_{k}^{k}(0,0)\right]\right\rangle .
$$

The holographic computations of the correlator (1.3) for a certain class of dual gravitational models by Gubser, Pufu and Rocha (GPR) was reported in [9]. It was claimed that some of the Einstein-scalar models considered led to a violation of the bound (1.1). On the other hand, in the Improved Holographic QCD model, [12-14], the bound (1.1) is comfortably obeyed, [31].

Recently an alternative expression for the bulk viscosity in strongly coupled plasmas with a holographic dual was obtained by Eling and $\mathrm{Oz}$ (EO) in [10]. They have analyzed directly the hydrodynamic limit of the equations of motion of a generic Einstein-scalar theory and derived a formula for the bulk viscosity that is apparently different from the GPR formula. The EO formula is very general and reads

$$
\frac{\zeta}{\eta}=\sum_{i}\left[s \frac{\partial \phi_{h}^{i}}{\partial s}+\sum_{a} \rho_{a} \frac{\partial \phi_{h}^{i}}{\partial \rho^{a}}\right]
$$

where $i$ labels different bulk scalars, $\phi_{h}^{i}$ is the value of the i-th scalar at the horizon, and $\rho^{a}$ are different conserved charged densities. The case comparable with GPR, involves a single scalar field and no charge density. In [10] the two formulae were shown to give the same result in cases where the adiabatic approximation to the equations is valid, but their equivalence in more general cases was put in doubt.

In this paper we re-analyze the bulk viscosity in two non-trivial holographic theories, the bosonic $\mathcal{N}=2^{*}$ theory [11] as well as Improved Holographic QCD, [12-15]. The bosonic $\mathcal{N}=2^{*}$ theory is $N=4$ superYM, with a non-trivial (and equal) mass for 4 of the 6 scalars. Improved holographic QCD on the other hand is a semi-phenomenological Einstein-scalar theory tuned to match non-supersymmetric Yang Mills theory in the large $N_{c}$ limit. $^{2}$

We point out that the analysis done in [9], when applied to $\mathcal{N}=2^{*}$ gauge theory plasma $[11,16-20]$ at high temperatures, agrees with earlier computations reported in $[1,10,21]$. The agreement is also checked numerically at all temperatures.

Both in the $\mathcal{N}=2^{*}$ theory and Improved Holographic QCD we confirm the agreement between GPR and EO formulae for the holographic bulk viscosity.

\footnotetext{
${ }^{2}$ Similar Einstein-scalar theories were also proposed to describe the crossover behavior of QCD with light quarks in [22].
} 


\section{The GPR formula for the holographic bulk viscosity}

Here we mostly follow [9]. For details we refer to the original work.

Consider a gravitational system, dual to some strongly coupled gauge theory plasma, described by an Einstein-dilaton system of the form

$$
S=\frac{1}{16 \pi G_{5}} \int d^{5} x \sqrt{-g}\left[R-\frac{1}{2}(\partial \phi)^{2}-V(\phi)\right] .
$$

The black brane background geometry dual to a thermal state of the plasma takes the form

$$
d s^{2} \equiv g_{\mu \nu}^{(0)} d x^{\mu} d x^{\nu}=e^{2 A(r)}\left[-h(r) d t^{2}+d \vec{x}^{2}\right]+e^{2 B(r)} \frac{d r^{2}}{h(r)}, \quad \phi=r .
$$

Notice that the field $\phi$ was chosen as a radial coordinate. ${ }^{3}$

The background equations of motion take a simple form

$$
\begin{aligned}
& 0=A^{\prime \prime}-A^{\prime} B^{\prime}+\frac{1}{6}, \\
& 0=h^{\prime \prime}+\left(4 A^{\prime}-B^{\prime}\right) h^{\prime}, \\
& 0=6 A^{\prime} h^{\prime}+h\left(24 A^{\prime 2}-1\right)+2 e^{2 B} V, \\
& 0=4 A^{\prime}-B^{\prime}+\frac{h^{\prime}}{h}-\frac{e^{2 B}}{h} V^{\prime} .
\end{aligned}
$$

To compute the correlation function (1.3), the authors of [9] considered an $\mathrm{SO}(3)$ invariant fluctuation of the metric $\delta g_{\mu \nu}(t, \phi) \rightarrow e^{-i \omega t} g_{\mu \nu}^{(0)}(\phi) H_{\mu \nu}(\phi)$ in the gauge $\delta \phi=0$. It was shown that the equation for $H_{11}$ decouples from the rest of the fluctuation equations and is ${ }^{4}$

$$
H_{11}^{\prime \prime}=\left(-\frac{1}{3 A^{\prime}}-4 A^{\prime}+3 B^{\prime}-\frac{h^{\prime}}{h}\right) H_{11}^{\prime}+\left(-\frac{e^{-2 A+2 B}}{h^{2}} \omega^{2}+\frac{h^{\prime}}{6 h A^{\prime}}-\frac{h^{\prime} B^{\prime}}{h}\right) H_{11} .
$$

One further has to solve (2.4) with the following UV $(r \rightarrow 0)$ and IR $\left(r \rightarrow \phi_{h}\right)^{5}$ boundary conditions:

$$
\begin{gathered}
\mathrm{UV}: \quad \lim _{r \rightarrow 0} H_{11}=1, \\
\mathrm{IR}: \quad H_{11} \rightarrow c_{11}^{-}\left(\phi_{h}-r\right)^{-i \omega / 4 \pi T}+0 \times\left(\phi_{h}-r\right)^{+i \omega / 4 \pi T}, \quad \text { as } \quad r \rightarrow \phi_{h} .
\end{gathered}
$$

The bulk viscosity, computed from (1.2), is given by [9]

$$
\frac{\zeta}{\eta}=\frac{1}{9 A^{\prime}\left(\phi_{h}\right)^{2}} \lim _{\omega \rightarrow 0}\left|c_{11}^{-}\right|^{2}
$$

\footnotetext{
${ }^{3}$ One might worry whether $\phi$ is monotonic from the boundary to the black brane horizon. In the Einstein-dilaton theory, there are solutions where $\phi^{\prime}$ vanishes along the flow. These where analyzed in [23] and shown to be unphysical, violating the Gubser bound [24]. Therefore, this is not expected to happen in the middle of an RG flow. At theories with an extra gauge field and at finite density however, it is possible that $\phi^{\prime}=0$ in a physical solution. A class of such examples were studied recently in [26].

${ }^{4}$ We independently reproduced this equation. We also verified the consistency of the gauge choice $\delta \phi=0$.

${ }^{5}$ Note that in the gauge we are working the position of the black hole horizon $r_{h}$ is identified with the value of the scalar at the horizon $\phi_{h}$.
} 
where one has to use the universality of the shear viscosity [5-8]. The authors of [9] used (2.3) to obtain

$$
A^{\prime}\left(\phi_{h}\right)=-\frac{V\left(\phi_{h}\right)}{3 V^{\prime}\left(\phi_{h}\right)},
$$

and arrived at the final formula for the bulk viscosity ratio

$$
\frac{\zeta}{\eta}=\frac{V^{\prime}\left(\phi_{h}\right)^{2}}{V\left(\phi_{h}\right)^{2}} \lim _{\omega \rightarrow 0}\left|c_{11}^{-}\right|^{2}
$$

\subsection{The EO versus GPR formula for the bulk viscosity}

In [10] Eling and Oz, by analyzing the hydrodynamic limit of the scalar-tensor equations, produced the following expression for the holographic bulk viscosity ${ }^{6}$

$$
\left.\frac{\zeta}{\eta}\right|_{\mathrm{EO}}=\left(s \frac{d \phi_{h}}{d s}\right)^{2}=\frac{1}{9 A^{\prime}\left(\phi_{h}\right)^{2}}
$$

Even though (up to a factor of $c_{11}^{-}$) (2.10) and (2.7) appear to be the same, they are, in fact, different: in (2.10),

$$
\left.A^{\prime}\left(\phi_{h}\right)\right|_{\mathrm{EO}}=\frac{d\left(\lim _{\phi \rightarrow \phi_{h}} A(\phi)\right)}{d \phi_{h}} \neq\left. A^{\prime}\left(\phi_{h}\right)\right|_{\mathrm{GPR}}=\lim _{\phi \rightarrow \phi_{h}} \frac{d A(\phi)}{d \phi}=\lim _{\phi \rightarrow \phi_{h}}-\frac{V(\phi)}{3 V^{\prime}(\phi)} .
$$

To be specific, in $\mathcal{N}=2^{*}$ gauge theory plasma at high temperature (see appendix A for some details)

$$
\begin{aligned}
\left.A^{\prime}\left(\phi_{h}\right)\right|_{\mathrm{EO}} & =\frac{\pi T^{2} \sqrt{6}}{m_{b}^{2}}+\mathcal{O}\left(\left(\frac{m_{b}^{2}}{T^{2}}\right)^{0}\right), \\
\left.A^{\prime}\left(\phi_{h}\right)\right|_{\mathrm{GPR}} & =\frac{2 \pi T^{2} \sqrt{6}}{m_{b}^{2}}+\mathcal{O}\left(\left(\frac{m_{b}^{2}}{T^{2}}\right)^{0}\right) .
\end{aligned}
$$

From (2.12) and (2.13) it is clear that (2.7) would produce the correct expression for the $\mathcal{N}=2^{*}$ plasma bulk viscosity, provided $^{7}$

$$
\left.\left|c_{11}^{-}\right|\right|_{\mathcal{N}=2^{*}, \text { prediction }}=2+\mathcal{O}\left(\frac{m_{b}^{2}}{T^{2}}\right) .
$$

In the next section we explicitly compute $c_{11}^{-}$, and find that it agrees with (2.14).

\section{Bulk viscosity calculation in the $\mathcal{N}=2^{*}$ plasma}

In this section we will first address the calculation of bulk viscosity in the $\mathcal{N}=2^{*}$ theory.

\footnotetext{
${ }^{6}$ The formula derived in [10] applies also to systems at finite charge density. Here we restrict our attention to zero charge density systems. The EO formula was further tested in [27].

${ }^{7}$ We assume the $\omega \rightarrow 0$ limit taken.
} 


\subsection{The computation of $c_{11}^{-}$in $x$-gauge}

We find it convenient to recast the equation for $H_{11}$ in terms of $x$ coordinate, defined as

$$
x \equiv 1-\sqrt{h} .
$$

Notice that $x \rightarrow 0_{+}$corresponds to the boundary and $x \rightarrow 1_{-}$to the horizon.

In this gauge the background equations take the form (all the derivatives are with respect to $x)$ :

$$
\begin{aligned}
& 0=A^{\prime \prime}-4\left(A^{\prime}\right)^{2}+\frac{A^{\prime}}{1-x}+\frac{1}{6}\left(\phi^{\prime}\right)^{2}, \\
& 0=\phi^{\prime \prime}-\frac{V_{, \phi}}{2 V}\left(\phi^{\prime}\right)^{2}+\frac{\phi^{\prime}}{x-1}+\frac{6 V_{, \phi} A^{\prime}\left(2 A^{\prime}(x-1)+1\right)}{V(1-x)},
\end{aligned}
$$

where

$$
V_{, \phi} \equiv \frac{d V}{d \phi} .
$$

The equation for $H_{11}$ is somewhat complicated

$$
0=H_{11}^{\prime \prime}+\mathcal{H}_{1} H_{11}^{\prime}+\mathcal{H}_{2} H_{11},
$$

where we collected the coefficients $\mathcal{H}_{i}$ in appendix B. In order to compute the bulk viscosity (2.7), we need to solve (3.5) subject to the following boundary conditions:

$$
\begin{aligned}
& \mathrm{UV}: \lim _{x \rightarrow 0_{+}} H_{11}=1 \text {, } \\
& \text { IR : } \quad H_{11} \rightarrow \tilde{c}_{11}^{-}(1-x)^{-i \omega / 2 \pi T}+0 \times(1-x)^{+i \omega / 2 \pi T}, \quad \text { as } \quad x \rightarrow 1_{-} .
\end{aligned}
$$

For generic $\omega, \tilde{c}_{11}^{-} \neq c_{11}^{-}$(see (2.6)), however, in the hydrodynamic limit

$$
\lim _{\omega \rightarrow 0} \tilde{c}_{11}^{-}=\lim _{\omega \rightarrow 0} c_{11}^{-} \equiv c_{11}^{-} .
$$

We can test (3.2)-(3.5) with a simple, exactly solvable background, like the exponential potential case. This is described in appendix C.

\section{$3.2 \mathcal{N}=2^{*}$ plasma at high temperatures}

The effective action of the gravitational dual to strongly coupled $\mathcal{N}=2^{*}$ plasma with a bosonic mass deformation is given by [20]

$$
S=\frac{1}{4 \pi G_{5}} \int_{\mathcal{M}_{5}} d \xi^{5} \sqrt{-g}\left[\frac{1}{4} R-3(\partial \alpha)^{2}-V\right],
$$

where the potential is ${ }^{8}$

$$
V=-\frac{1}{4} e^{-4 \alpha}-\frac{1}{2} e^{2 \alpha} .
$$

\footnotetext{
${ }^{8}$ We set the five-dimensional gauged supergravity coupling to one. This corresponds to setting the radius $\ell$ of the five-dimensional sphere in the undeformed metric to 2 .
} 
Notice that the canonically normalized scalar is $\phi=\sqrt{24} \alpha$, and therefore

$$
V_{, \phi}=\frac{1}{\sqrt{24}} V_{, \alpha} .
$$

We will study the theory (3.9) in the high-temperature regime. In this case (see appendix A)

$$
\begin{aligned}
e^{\alpha} & \equiv \rho=1+\delta_{1} \alpha_{1}+\mathcal{O}\left(\delta_{1}^{2}\right), \\
A & =\ln \delta_{3}-\frac{1}{4} \ln \left(2 x-x^{2}\right)+\delta_{1}^{2} A_{1}+\mathcal{O}\left(\delta_{1}^{4}\right) .
\end{aligned}
$$

In the hydrodynamic limit, i.e., $\omega \rightarrow 0$, and to leading order in $\delta_{1}$, we find

$$
\begin{aligned}
0+\mathcal{O}\left(\delta_{1}\right)= & H_{11}^{\prime \prime}+\frac{\left(x \alpha_{1}^{\prime}(2-x)\left(x^{2}-2 x+4\right)-2 \alpha_{1}(1-x)\right.}{\alpha_{1}^{\prime} x^{2}(1-x)(2-x)^{2}} H_{11}^{\prime} \\
& +\frac{2\left(\left(4 x-2 x^{2}\right) \alpha_{1}^{\prime}+\alpha_{1}(x-1)\right)}{(2-x)^{2}(1-x)^{2} \alpha_{1}^{\prime} x^{2}} H_{11} .
\end{aligned}
$$

Notice that there is dependence only on $\alpha_{1}$, which satisfied the following equation

$$
0=\alpha_{1}^{\prime \prime}+\frac{1}{x-1} \alpha_{1}^{\prime}+\frac{1}{x^{2}(2-x)^{2}} \alpha_{1} .
$$

Even though we know an analytic solution for $\alpha_{1}$ (see (A.2)), we can not solve for $H_{11}$ analytically. We find it convenient to use numerical techniques to solve both (3.13) and (3.14). Near the boundary we have

$$
\alpha_{1}=\sqrt{x}\left(\sum_{n=0}^{\infty} \sum_{k=0}^{1} a_{n, k} x^{n} \ln ^{k} x\right),
$$

with normalization ${ }^{9} a_{0,1}=1$, and

$$
a_{n, k}=a_{n, k}\left(a_{0,0}\right) .
$$

For example, for the first few terms we have:

$$
a_{1,0}=\frac{1}{2}+\frac{1}{4} a_{0,0}, \quad a_{1,1}=\frac{1}{4}, \quad a_{2,0}=\frac{5}{16}+\frac{5}{32} a_{0,0}, \quad a_{2,1}=\frac{5}{32} .
$$

The asymptotic expansion for $H_{11}$ is a bit unusual because the perturbing operator has scaling dimension 2 :

$$
H_{11}=\sum_{n=0}^{\infty} \sum_{k=0}^{n+1} h_{n, k} x^{n} \frac{1}{\left(a_{0,0}+2+\ln x\right)^{k}},
$$

with normalization $h_{0,0}=1$, see (3.6). Here,

$$
h_{n, k}=h_{n, k}\left(h_{0,1}\right) \text {. }
$$

\footnotetext{
${ }^{9}$ The overall normalization of $\alpha_{1}$ is arbitrary, we choose the leading $\ln x$ coefficient to be 1 .
} 
For the first few terms we have:

$$
\begin{aligned}
& h_{1,0}=-1, \quad h_{1,1}=-h_{0,1}, \quad h_{1,2}=-\frac{1}{2} h_{0,1}, \\
& h_{2,0}=-\frac{1}{4}, \quad h_{2,1}=\frac{1}{4}\left(1-h_{0,1}\right), \quad h_{2,2}=\frac{9}{16} h_{0,1}, \quad h_{2,3}=\frac{1}{4} h_{0,1} .
\end{aligned}
$$

Near the horizon, $y \equiv 1-x$, we obtain

$$
\begin{aligned}
\alpha_{1} & =a^{h} \sum_{n=0}^{\infty} a_{n}^{h} y^{2 n}=a^{h}\left(1-\frac{1}{4} y^{2}-\frac{7}{64} y^{4}-\frac{17}{256} y^{6}+\cdots\right), \\
H_{11} & =h^{h} \sum_{n=0}^{\infty} h_{n}^{h} y^{2 n}=h^{h}\left(1-\frac{1}{8} y^{2}-\frac{3}{64} y^{4}-\frac{27}{1024} y^{6}+\cdots\right) .
\end{aligned}
$$

Altogether we have four integration constants:

$$
\left\{a_{0,0}, h_{0,1}, a^{h}, h^{h}\right\},
$$

precisely what is needed to solve uniquely the system of two second order ODEs: (3.13) and (3.14). Using numerical techniques developed in [28] we find

$$
\begin{array}{ll}
a_{0,0}=-2.079441(5), & a^{h}=-2.221441(5), \\
h_{0,1}=-2.000000(0), & h^{h}=2.000000(0) .
\end{array}
$$

Of course, $\left\{a_{0,0}, a^{h}\right\}$ are known analytically from (A.2),

$$
\left\{a_{0,0}, a^{h}\right\}=\left\{-\ln 8,-\frac{\pi}{\sqrt{2}}\right\}
$$

and are in excellent agreement with (3.22).

From (3.22),

$$
\left.c_{11}^{-}\right|_{\mathcal{N}=2 *}=h^{h}=2,
$$

to a very good accuracy, confirming the agreement of bulk viscosity for the hightemperature $\mathcal{N}=2^{*}$ plasma from (2.7) with earlier computations $[1,10,21]$.

\section{3 $\mathcal{N}=2^{*}$ plasma at generic temperatures for $\frac{m_{b}^{2}}{T^{2}}>0$}

It is straightforward to extend the analysis of the previous section to generic temperatures $\mathcal{N}=2^{*}$ gauge theory plasma for physical mass deformations, i.e., $\frac{m_{b}^{2}}{T^{2}}>0$. The background geometry was studied in [20], and the bulk viscosity (from the sound waves dispersion relation) was computed in [4]. The results of the analysis are reported in figure 1 . We further verified that the GPR formula (2.9) for the bulk viscosity, when applied to $\mathcal{N}=2^{*}$ plasma, agrees with the bulk viscosity of the theory at criticality [4] computed from the sound waves dispersion relation to $\approx 5 \times 10^{-7}$.

\section{Bulk viscosity calculation in improved holographic QCD}

In this section we perform an independent calculation of the coefficient $c_{11}^{-}$by the methods developed in $[23,31]$. As described in section 7 of [23], one can work out the thermodynamics of gravity-scalar system entirely by solving a system of coupled first order equations for the so-called phase variables introduced below. 


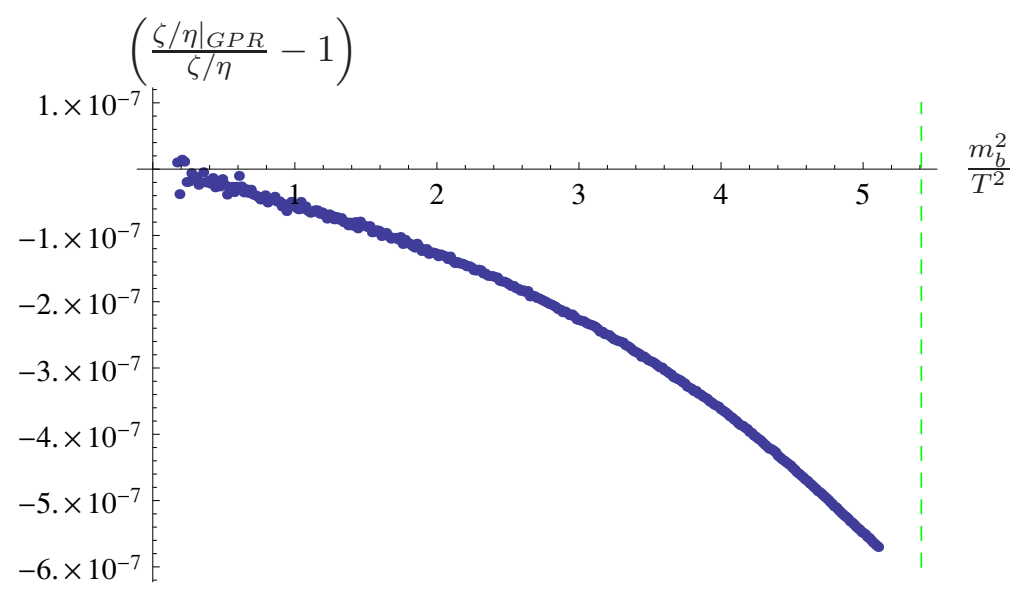

Figure 1. (Color online) Comparison of the GPR prediction for $\mathcal{N}=2^{*}$ plasma bulk viscosity with the explicit computations from the quasinormal modes [4]. The dashed vertical green line represents the critical point of the theory $\frac{m_{b}^{2}}{T^{2}}=\delta_{c}=5.4098(6)$ associated with the second-order phase transition $[29,30]$.

\subsection{Computation of $c_{11}^{-}$using phase variables}

Starting with the action (2.1) we look for a black-hole solution of the form,

$$
d s^{2}=e^{2 A(r)}\left(h^{-1}(r) d r^{2}+d x_{d-1}^{2}+d t^{2} h(r)\right), \quad \phi=\phi(r) .
$$

We are interested in solutions that are asymptotically AdS. In the dual field theory this corresponds to the presence of conformal invariance in the UV that is broken either explicitly by a mass deformation as in the $\mathcal{N}=2^{*}$ theory or by a marginal deformation as in the phenomenological models of [12-14].

In the gauge $\delta \phi=0$ one can equivalently use $\phi$ as the radial variable. Defining the following phase variables [12-14, 23],

$$
X(\phi) \equiv \frac{1}{4} \sqrt{\frac{2}{3}} \frac{\phi^{\prime}}{A^{\prime}}, \quad Y(\phi) \equiv \frac{1}{4} \frac{h^{\prime}}{h A^{\prime}},
$$

the Einstein's equations can be reduced to

$$
\begin{aligned}
& \frac{d X}{d \phi}=-\sqrt{\frac{2}{3}}\left(1-X^{2}+Y\right)\left(1+\sqrt{\frac{3}{8}} \frac{1}{X} \frac{d \log V}{d \phi}\right), \\
& \frac{d Y}{d \phi}=-\sqrt{\frac{2}{3}}\left(1-X^{2}+Y\right) \frac{Y}{X} .
\end{aligned}
$$

This coupled first order system is sufficient to determine all of the thermodynamic properties (and dissipation) of the gravitational theory [23]. Once a solution to (4.3), (4.4) is constructed, the metric functions can be determined as,

$$
\begin{aligned}
A(\phi) & =A_{0}+\frac{1}{4} \sqrt{\frac{2}{3}} \int_{\phi_{0}}^{\phi} \frac{1}{X} d \tilde{\phi}, \\
h(\phi) & =\exp \left(\sqrt{\frac{2}{3}} \int_{\phi_{0}}^{\phi} \frac{Y}{X} d \tilde{\phi}\right) .
\end{aligned}
$$


Here $\phi_{0}$ corresponds to the UV value at the boundary corresponding to the UV AdS minimum of the potential. $A_{0}$ is an integration constant that essentially determines the energy scale of the breaking of conformal symmetry.

The thermodynamics of the black-hole can directly be determined as follows. The free energy is given by

$$
F\left(\phi_{h}\right)=\frac{1}{4 G_{5}} \int_{\phi_{h}}^{\infty} d \tilde{\phi}_{h} e^{3 A\left(\phi_{h}\right)} \frac{d T}{d \tilde{\phi}_{h}} .
$$

These backgrounds satisfy the 1st law of thermodynamics $S=-d F / d T$. Equation (4.7) directly follows from integrating this equation, where

$$
S=\frac{1}{4 \pi G_{5}} e^{3 A\left(\phi_{h}\right)}
$$

is used. In the integration in (4.7) one should make sure that the UV asymptotics is kept fixed as $\phi_{h}$ is varied. This is explained in the case of marginal deformations in section 7 of $[23]$.

The temperature as a function of $\phi_{h}$ is obtained from

$$
T\left(\phi_{h}\right)=\frac{\ell}{12 \pi} e^{A\left(\phi_{h}\right)} V\left(\phi_{h}\right) e^{\sqrt{\frac{2}{3}} \int_{\phi_{0}}^{\phi_{h}} X(\phi) d \phi} .
$$

Once we solve (4.3) and (4.4) above, we can calculate the free energy as a function of $A_{0}$ and $T$ by employing the formulae above.

\subsection{The fluctuation equation}

The fluctuation equation (2.4) in terms of the phase variables read,

$$
H_{11}^{\prime \prime}=c(\phi) H_{11}^{\prime}+d(\phi) H_{11}
$$

where

$$
\begin{aligned}
& c(\phi)=\frac{1-X^{2}+Y}{X}\left(\frac{4}{\sqrt{6}}+\frac{3}{2 X} \frac{V^{\prime}}{V}\right) \\
& d(\phi)=-\frac{2 Y}{3 X^{2}}\left(1-X^{2}+Y\right)\left(1+\frac{\sqrt{3}}{\sqrt{8} X} \frac{V^{\prime}}{V}\right)-\left(\frac{2}{3} \frac{\omega Y}{4 \pi T X}\right)^{2} e^{-\sqrt{\frac{3}{2}} \int_{\phi}^{\phi_{h}} \frac{1}{X}} .
\end{aligned}
$$

In passing, we note that changing the variable back to the original radial coordinate in (4.1) produces a rather simple equation [25]:

$$
\ddot{H}_{11}+\dot{H}_{11}\left(3 \dot{A}+\frac{\dot{h}}{h}+\mathbf{2} \frac{\dot{\mathbf{X}}}{\mathbf{X}}\right)+\dot{H}_{11}\left(\frac{\omega_{h}^{2}}{h^{2}}-\frac{\dot{\mathbf{h}}}{\mathbf{h}} \frac{\dot{\mathbf{X}}}{\mathbf{X}}\right)=0,
$$

where we emphasized the new terms in the bulk fluctuation eq. that arise from mixing of the rotationally invariant graviton excitations and the dilaton. The normalized frequency is defined by $\omega_{h}=\omega r_{h}$. This equation compares with the one corresponding to the shear fluctuations:

$$
\ddot{H}_{12}+\dot{H}_{12}\left(3 \dot{A}+\frac{\dot{h}}{h}\right)+H_{12} \frac{\omega_{h}^{2}}{h^{2}}=0 .
$$


One crucial difference between (4.13) and (4.14) is that, unlike in the case of the shear deformation, the bulk deformation has a mass term even in the hydrodynamic limit $\omega_{h}=0$. This implies that in general there should be a non-trivial flow from the horizon to the boundary in the sense of the membrane paradigm [32]. This flow is absent only in the case $X=$ const which corresponds to the adiabatic limit [31]. It is also absent in the Chamblin-Reall solution that corresponds to constant $X$, see section $\mathrm{C}$.

In the following we apply the formalism developed here to calculate the bulk viscosity in two examples.

\subsection{Numerical results for the holographic-QCD model}

As another non-trivial example, we would like to confirm the agreement between the EO and the GPR formula in the improved holographic QCD model of [12-14]. The model is based on a single scalar in the bulk theory corresponding to the operator $\operatorname{Tr} F^{2}$ in the $\mathrm{SU}(N)$ gauge theory. Therefore the deformation in the UV is marginally relevant, hence the UV asymptotics is not of the standard asymptotically AdS type, but involves logarithmic corrections. In the following we present the results in the variable

$$
\lambda=e^{\sqrt{\frac{3}{8}} \phi} .
$$

The scalar potential is given by,

$$
V(\lambda)=-\frac{12}{\ell^{2}}\left\{1+V_{0} \lambda+V_{1} \lambda^{4 / 3}\left[\log \left(1+V_{2} \lambda^{4 / 3}+V_{3} \lambda^{2}\right)\right]^{1 / 2}\right\}
$$

The various parameters in (4.16)

$$
\left\{V_{0}, V_{1}, V_{2}, V_{3}\right\}=\left\{0.0413,14,5.310^{-9}, 170\right\},
$$

are fixed in order to fit the UV asymptotics of $\mathrm{SU}(N)$ beta-function, the observed latent heat of the confinement-deconfinement transition on the lattice and the agreement with the glueball spectrum in the vacuum theory [33].

A straightforward application of the method explained in section 4.1 yields the bulk viscosity from the GPR formula [31]. In the figure 2 we compare the outcome of the two formulae for a range of $\lambda_{h}$ that corresponds to the entire range of temperatures above the deconfinement transition, $T>T_{c}$ on the thermodynamically favored and stable big black-hole branch. As seen from this figure the two formulae match to great accuracy.

\section{Acknowledgments}

We would like to thank Yaron $\mathrm{Oz}$ for valuable correspondence and discussions. Research at Perimeter Institute is supported by the Government of Canada through Industry Canada and by the Province of Ontario through the Ministry of Research \& Innovation. AB gratefully acknowledges further support by an NSERC Discovery grant and support through the Early Researcher Award program by the Province of Ontario. This work was partially supported by European Union grants FP7-REGPOT-2008-1-CreteHEPCosmo-228644, and PERG07-GA-2010-268246 . 


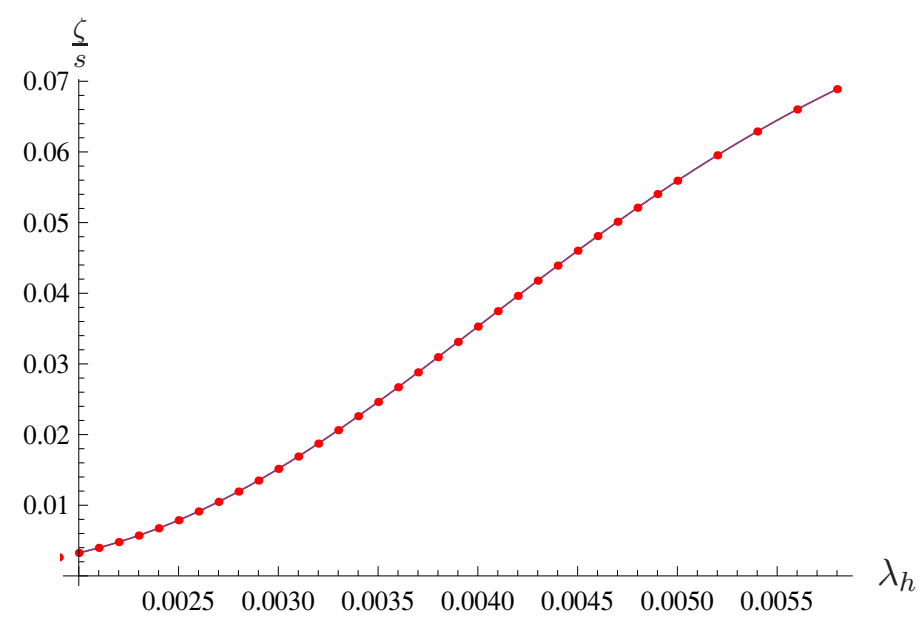

Figure 2. Comparison of the bulk viscosity in the phenomenological QCD model. The solid (blue) curve represents the outcome of the Eling-Oz formula. The (red) dots are the outcome of Gubser et al's formula.

\section{A $\quad \frac{d A}{d \phi}$ in $\mathcal{N}=2^{*}$ plasma}

From [20], to leading order in $m_{b}^{2} / T^{2}$ (notice the $\sqrt{24}$ renormalization of the $\alpha$ to insure the canonical kinetic term as in (2.1)),

$$
\begin{aligned}
& A(x)=\ln \delta_{3}-\frac{1}{4} \ln \left(2 x-x^{2}\right)+\delta_{1}^{2} A_{1}(x), \\
& \phi(x)=\sqrt{24} \delta_{1} \alpha_{1}(x)
\end{aligned}
$$

where

$$
\begin{aligned}
& \alpha_{1}=\left(2 x-x^{2}\right)_{2}^{1 / 2} F_{1}\left(\frac{1}{2}, \frac{1}{2} ; 1 ;(1-x)^{2}\right), \\
& A_{1}=4 \int_{x}^{1} \frac{(z-1) d z}{\left(2 z-z^{2}\right)^{2}}\left(\gamma_{1}-\int_{z}^{1} d y\left(\frac{\partial \alpha_{1}}{\partial y}\right)^{2} \frac{\left(2 y-y^{2}\right)^{2}}{y-1}\right), \\
& \gamma_{1}=\frac{8-\pi^{2}}{2 \pi^{2}}, \quad 2 \pi T=\delta_{3}\left(1+\frac{16}{\pi^{2}} \delta_{1}^{2}\right), \quad \delta_{1}=-\frac{1}{24 \pi}\left(\frac{m_{b}}{T}\right)^{2} .
\end{aligned}
$$

From (A.1)-(A.4) it is easy to deduce that near the horizon, i.e., $x \rightarrow 1_{-}$,

$$
\begin{aligned}
& \phi=\phi_{h}\left(1-\frac{1}{4}(1-x)^{2}+\mathcal{O}\left((1-x)^{4}\right)\right), \quad \phi_{h}=-\frac{m_{b}^{2}}{2 \sqrt{6} \pi T^{2}} \\
& A=\ln (2 \pi T)-\frac{m_{b}^{4}}{36 \pi^{4} T^{4}}+\left(\frac{1}{4}-\frac{\gamma_{1} m_{b}^{4}}{288 \pi^{2} T^{4}}\right)(1-x)^{2}+\mathcal{O}\left((1-x)^{4}\right) .
\end{aligned}
$$

We can now compute $\frac{d A}{d \phi}$ while keeping $m_{b}$ fixed, see (2.12) and (2.13). 


\section{B Coefficients $\mathcal{H}_{i}$}

In the appendix we explicitly show the coefficients of the fluctuation equation (3.5):

$$
\begin{aligned}
\mathcal{H}_{1}= & \frac{\left(\phi^{\prime 2}(x-1)-12 A^{\prime}\left(2(x-1) A^{\prime}+1\right)\right) V_{, \phi}}{(x-1) \phi^{\prime} V}+\frac{\phi^{\prime 2}(x-1)-3 A^{\prime}\left(3+8(x-1) A^{\prime}\right)}{3(x-1) A^{\prime}}, \\
\mathcal{H}_{2}= & \frac{\phi^{\prime 2}(x-1)-12 A^{\prime}\left(2(x-1) A^{\prime}+1\right)}{6(x-1)^{3} \phi^{\prime} V A^{\prime}} \times\left(2 \phi^{\prime} V(1-x)+6 V_{, \phi}(1-x) A^{\prime}\right. \\
& \left.+3 e^{-2 A} \omega^{2} \phi^{\prime} A^{\prime}\right) .
\end{aligned}
$$

\section{Chamblin-Reall backgrounds}

We choose an exponential potential (known also as the Chamblin-Reall geometry), [34]. In this case

$$
V=\mathcal{V} e^{g \phi}
$$

with constant $\mathcal{V}$.

Solving the background equations we find,

$$
\begin{aligned}
A(x) & =\mathcal{A}-\frac{1}{3 g} \phi(x), \\
\exp \left(\frac{3 g^{2}-8}{6 g} \phi(x)\right) & =\frac{1}{\mathcal{P}(x-1)^{2}+1-\mathcal{P}},
\end{aligned}
$$

where $\mathcal{A}, \mathcal{P}$ are the integration constants, and without the loss of generality we assumed $\phi(0)=0$.

To leading order in the hydrodynamic limit (3.5) simplifies dramatically

$$
0=H_{11}^{\prime \prime}+\frac{1}{x-1} H_{11}^{\prime} .
$$

We outline now the solution of the boundary value problem that we will use in the more complicated example of the $\mathcal{N}=2^{*}$ gauge theory below.

First, the general solution with the UV boundary condition (3.6) is given by

$$
H_{11}^{b}=1+h_{\mathrm{uv}} \ln (1-x),
$$

with an arbitrary constant $h_{\mathrm{uv}}$. It is straightforward to rewrite (C.3) in terms of $y=1-x$, and find the most general solution satisfying the IR boundary condition (3.7)

$$
H_{11}^{h}=h_{\text {ir }},
$$

with an arbitrary constant $h_{i r}$. Matching $H_{11}^{b}$ and $H_{11}^{h}$ (the value of the function and the first derivatives) uniquely determines

$$
h_{\mathrm{uv}}=0, \quad h_{\mathrm{ir}}=1 .
$$


Thus, much like in [9], we conclude that for the Chamblin-Reall model

$$
c_{11}^{-}=1
$$

We may also present the results above in the language of phase variables, (4.2). The Chamblin-Reall solution is given by (see appendix $\mathrm{J}$ of [23]),

$$
X=-\sqrt{\frac{3}{8}} g, \quad Y=\frac{1-X^{2}}{e^{\alpha\left(\phi_{h}-\phi\right)}-1},
$$

where we defined the constant,

$$
\alpha=-\sqrt{\frac{2}{3}} \frac{\left(1-X^{2}\right)}{X} .
$$

We note that for consistency of thermodynamics $X^{2}<1$, otherwise the black-hole solution has negative specific heat, hence corresponds to a small black-hole [23]. One finds the following metric functions in the variable $\phi$ :

$$
\begin{gathered}
A(\phi)=A_{0}+\frac{1}{\sqrt{6} X} \phi, \\
h(\phi)=1-e^{\alpha\left(\phi-\phi_{h}\right)} .
\end{gathered}
$$

One distinguishing fact about the above solution is that the scale function $A(\phi)$ is independent of the temperature $\phi_{h}$.

Before making this connection however, let us provide a simple proof - closely related to the one given in section C. The fluctuation equation (4.10) simplifies drastically as the coefficient $d(\phi)$ in (4.12) vanishes for $\omega=0$. This means in particular that there is no flow from the horizon to the boundary in the sense of the membrane paradigm, see e.g. [32] for the bulk-viscosity in the case of Chamblin-Reall backgrounds.

The proof that $\left|c_{11}^{-}\right|\left(\phi_{h}\right)=1$ in this case is already given in appendix B of [31], that we review here. When, the coefficient $d(\phi)$ in (4.12) vanishes in the $\omega=0$ equation, the solution to $H_{11}$ is simply given by,

$$
H_{11}(\phi)=1+C \int_{-\phi_{0}}^{\phi} d t e^{\int_{-\phi_{0}}^{t} c(t)}
$$

where the function $c(\phi)$ is given by (4.11) and we used the boundary condition $H_{11}\left(\phi_{0}\right)=1$. The second integration constant $C$ is determined by the second boundary condition that $H_{11}(\phi)$ is regular at the horizon [9]. On the other hand, the function $c(\phi)$ in (4.11) is positive definite because $X<0, Y>0, X^{2}<1$, and the term inside the brackets is given by $-4 / 3 g$, hence negative. Therefore the only way to guarantee regularity at the horizon is to set $C=0$, hence $H_{11}=1$ for all values of $\lambda$ in the limit, in particular $\left|c_{11}^{-}\right|$is 1 .

Open Access. This article is distributed under the terms of the Creative Commons Attribution Noncommercial License which permits any noncommercial use, distribution, and reproduction in any medium, provided the original author(s) and source are credited. 


\section{References}

[1] P. Benincasa, A. Buchel and A.O. Starinets, Sound waves in strongly coupled non-conformal gauge theory plasma, Nucl. Phys. B 733 (2006) 160 [hep-th/0507026] [SPIRES].

[2] J.M. Maldacena, The large $N$ limit of superconformal field theories and supergravity, Adv. Theor. Math. Phys. 2 (1998) 231 [Int. J. Theor. Phys. 38 (1999) 1113] [hep-th/9711200] [SPIRES].

[3] O. Aharony, S.S. Gubser, J.M. Maldacena, H. Ooguri and Y. Oz, Large N field theories, string theory and gravity, Phys. Rept. 323 (2000) 183 [hep-th/9905111] [SPIRES].

[4] A. Buchel, Bulk viscosity of gauge theory plasma at strong coupling, Phys. Lett. B 663 (2008) 286 [arXiv:0708.3459] [SPIRES].

[5] A. Buchel and J.T. Liu, Universality of the shear viscosity in supergravity, Phys. Rev. Lett. 93 (2004) 090602 [hep-th/0311175] [SPIRES].

[6] P. Kovtun, D.T. Son and A.O. Starinets, Viscosity in strongly interacting quantum field theories from black hole physics, Phys. Rev. Lett. 94 (2005) 111601 [hep-th/0405231] [SPIRES].

[7] A. Buchel, On universality of stress-energy tensor correlation functions in supergravity, Phys. Lett. B 609 (2005) 392 [hep-th/0408095] [SPIRES].

[8] P. Benincasa, A. Buchel and R. Naryshkin, The shear viscosity of gauge theory plasma with chemical potentials, Phys. Lett. B 645 (2007) 309 [hep-th/0610145] [SPIRES].

[9] S.S. Gubser, S.S. Pufu and F.D. Rocha, Bulk viscosity of strongly coupled plasmas with holographic duals, JHEP 08 (2008) 085 [arXiv:0806.0407] [SPIRES].

[10] C. Eling and Y. Oz, A Novel Formula for Bulk Viscosity from the Null Horizon Focusing Equation, JHEP 06 (2011) 007 [arXiv:1103.1657] [SPIRES].

[11] K. Pilch and N.P. Warner, $N=2$ supersymmetric $R G$ flows and the IIB dilaton, Nucl. Phys. B 594 (2001) 209 [hep-th/0004063] [SPIRES].

[12] U. Gürsoy and E. Kiritsis, Exploring improved holographic theories for QCD: Part I, JHEP 02 (2008) 032 [arXiv:0707.1324] [SPIRES].

[13] U. Gürsoy, E. Kiritsis and F. Nitti, Exploring improved holographic theories for QCD: Part II, JHEP 02 (2008) 019 [arXiv:0707.1349] [SPIRES].

[14] U. Gürsoy, E. Kiritsis, L. Mazzanti, G. Michalogiorgakis and F. Nitti, Improved Holographic QCD, Lect. Notes Phys. 828 (2011) 79 [arXiv:1006.5461] [SPIRES].

[15] U. Gürsoy, E. Kiritsis, L. Mazzanti and F. Nitti, Deconfinement and Gluon Plasma Dynamics in Improved Holographic QCD, Phys. Rev. Lett. 101 (2008) 181601 [arXiv:0804.0899] [SPIRES].

[16] A. Buchel, A.W. Peet and J. Polchinski, Gauge dual and noncommutative extension of an $N=2$ supergravity solution, Phys. Rev. D 63 (2001) 044009 [hep-th/0008076] [SPIRES].

[17] N.J. Evans, C.V. Johnson and M. Petrini, The enhancon and $N=2$ gauge theory/gravity $R G$ flows, JHEP 10 (2000) 022 [hep-th/008081] [SPIRES].

[18] A. Buchel and J.T. Liu, Thermodynamics of the $N=2^{*}$ flow, JHEP 11 (2003) 031 [hep-th/0305064] [SPIRES]. 
[19] A. Buchel, $N=2^{*}$ hydrodynamics, Nucl. Phys. B 708 (2005) 451 [hep-th/0406200] [SPIRES].

[20] A. Buchel, S. Deakin, P. Kerner and J.T. Liu, Thermodynamics of the $N=2^{*}$ strongly coupled plasma, Nucl. Phys. B 784 (2007) 72 [hep-th/0701142] [SPIRES].

[21] A. Yarom, Notes on the bulk viscosity of holographic gauge theory plasmas, JHEP 04 (2010) 024 [arXiv:0912.2100] [SPIRES].

[22] S.S. Gubser and A. Nellore, Mimicking the QCD equation of state with a dual black hole, Phys. Rev. D 78 (2008) 086007 [arXiv:0804.0434] [SPIRES].

[23] U. Gürsoy, E. Kiritsis, L. Mazzanti and F. Nitti, Holography and Thermodynamics of $5 D$ Dilaton-gravity, JHEP 05 (2009) 033 [arXiv: 0812.0792] [SPIRES].

[24] S.S. Gubser, Curvature singularities: The good, the bad and the naked, Adv. Theor. Math. Phys. 4 (2000) 679 [hep-th/0002160] [SPIRES].

[25] U. Gürsoy, Continuous Hawking-Page transitions in Einstein-scalar gravity, JHEP 01 (2011) 086 [arXiv:1007.0500] [SPIRES].

[26] C. Charmousis, B. Gouteraux, B.S. Kim, E. Kiritsis and R. Meyer, Effective Holographic Theories for low-temperature condensed matter systems, JHEP 11 (2010) 151 [arXiv: 1005 .4690] [SPIRES].

[27] A. Buchel, On Eling-Oz formula for the holographic bulk viscosity, JHEP 05 (2011) 065 [arXiv:1103.3733] [SPIRES].

[28] O. Aharony, A. Buchel and P. Kerner, The black hole in the throat - thermodynamics of strongly coupled cascading gauge theories, Phys. Rev. D 76 (2007) 086005 [arXiv:0706.1768] [SPIRES].

[29] A. Buchel and C. Pagnutti, Transport at criticality, Nucl. Phys. B 834 (2010) 222 [arXiv: 0912.3212] [SPIRES].

[30] A. Buchel and C. Pagnutti, Critical phenomena in $N=2^{*}$ plasma, Phys. Rev. D 83 (2011) 046004 [arXiv:1010.3359] [SPIRES].

[31] U. Gürsoy, E. Kiritsis, G. Michalogiorgakis and F. Nitti, Thermal Transport and Drag Force in Improved Holographic QCD, JHEP 12 (2009) 056 [arXiv:0906.1890] [SPIRES].

[32] N. Iqbal and H. Liu, Universality of the hydrodynamic limit in AdS/CFT and the membrane paradigm, Phys. Rev. D 79 (2009) 025023 [arXiv:0809.3808] [SPIRES].

[33] U. Gürsoy, E. Kiritsis, L. Mazzanti and F. Nitti, Improved Holographic Yang-Mills at Finite Temperature: Comparison with Data, Nucl. Phys. B 820 (2009) 148 [arXiv: 0903.2859] [SPIRES].

[34] H.A. Chamblin and H.S. Reall, Dynamic dilatonic domain walls, Nucl. Phys. B 562 (1999) 133 [hep-th/9903225] [SPIRES]. 